\title{
Redistribution of nutritionally important starch fractions beneficial to diabetic population by using oats as a functional ingredient in a wheat-based food product - Jereesh
}

\author{
Faiyaz Ahmed
}

\author{
Department of Clinical Nutrition, College of Applied Health Sciences in Ar Rass, Qassim University, Al Qassim Region 51921, Saudi \\ Arabia. Tel: +966163010496; E-mail: f.masfoor@qu.edu.sa \\ DOI: $10.31665 /$ JFB.2021.15284 \\ Received: July 10, 2021; Revised received \& accepted: September 28, 2021 \\ Citation: Ahmed, F. (2021). Redistribution of nutritionally important starch fractions beneficial to diabetic population by using oats as a \\ functional ingredient in a wheat-based food product - Jereesh. J. Food Bioact. 15: 74-79.
}

\begin{abstract}
Oats being important sources of $\beta$-glucan were incorporated at 10, 20 and $30 \%$ level in a wheat-based traditional Arabian breakfast cereal called Jereesh and evaluated for sensory acceptability. Sensory analysis reflected that the products with 10 and $20 \%$ oats incorporation were acceptable in terms of color, appearance, taste, aroma, mouthfeel, after taste and overall quality as indicated by the sensory scores which revealed no substantial differences between control jereesh (CJ) and oats incorporated jereesh (OJ). However, jereesh with $30 \%$ oats incorporation was very sticky (viscous) and scored low in sensory evaluation. Thus, nutritionally important starch fractions were measured in jereesh with $20 \%$ oats incorporation using a controlled in vitro multi-enzymatic method. Results indicated that, addition of oats at $20 \%$ level resulted in a substantial decrease $(p<0.05)$ in total starch (TS), rapidly available glucose (RAG), and resistant starch content (RS), as well as a significant increase $(p<0.05)$ in slowly digestible starch (SDS) compared to the control jereesh. The starch digestibility index remained unchanged. The findings of the study suggest that incorporation of oats helps in value addition of jereesh in terms of slow digestibility with increased soluble dietary fiber content that might lower glycemic index.
\end{abstract}

Keywords: Jereesh; Oats; Sensory analysis; Starch digestibility; Starch fractions.

\section{Introduction}

Cereal-based foods containing starch as the principal component form a major source of energy worldwide particularly in traditional diets. Nutritionally, distribution of starch fractions namely, rapidly digestible starch (RDS, hydrolyzed within $20 \mathrm{~min}$ ), slowly digestible starch (SDS, hydrolyzed after $20 \mathrm{~min}$ but before $120 \mathrm{~min}$ ), and resistant starch (RS, remain unhydrolyzed even after $120 \mathrm{~min}$ ) which determine digestibility of starchy foods is of particular importance in populations with high prevalence of metabolic diseases (Englyst et al., 1992; Samarakoon, 2020).

Jereesh is one such wheat-based traditional food product with higher gelatinization of starch due to the sheer amount of water used in its preparation (Al-Faris, 2017). Traditionally, jereesh is made with whole grains and chicken, wherein a single serving of $150 \mathrm{~g}$ provides $16 \%$ of the RDA for fiber. As a low glycemic index food (GI: 52), it is believed that jereesh intake can make a substantial contribution to the improvement of Saudi diets. However, in recent times consumption of jereesh is reduced and processed grains have now replaced whole grains diminishing the low GI advantage of jereesh (Nasib, 2003; Al-Mssallem, 2014). Several studies on cereal starches have shown that starch digestibility is affected by both internal and extrinsic parameters, and the degree of gelatinization, which increases with water and cooking time, is one such factor that has a significant impact on starch digestibility because of the altered susceptibility of cereal starches to enzymatic breakdown (Englyst et al., 1992; Samarakoon, 2020; Samarakoon et al., 2020). Studies have shown that, the inclusion of certain food components or modifications in processing conditions might result in redistribution of 
starch fractions, opening the door to the production of tailored food products for different health conditions (Giri et al., 2017).

Oats are an example of a functional ingredient that has considerable potential for use in the formulation of functional foods. Their use, however, is restricted to porridges and other plain breakfast cereals (Butt et al., 2008). More recently, interest in using oats in food product development has increased, not only because of the presence of high amounts of $\beta$-glucans, which have a lipid lowering/plasma glucose stabilizing effect, but also because of the unique gelatinization characteristics of its starch, which behaves like a waxy starch due to high shear susceptibility (Peterson, 2004). In comparison to other cereal starches, the high viscosity starch gel thus generated form oats is less prone to retrogradation upon cooling, producing an elastic, sticky, and less rigid gel (Wang and White, 1994). These properties make oats starch appropriate for use in the formulation of functional foods. It is worth noting that adding oats into food products not only improves soluble dietary fiber content, which has lipid-lowering and plasma glucose-stabilizing effects, but also provides minor phytochemicals, which have health benefits including improved gut health (Harasym and Olędzki, 2018). Addition of oats will also substantially reduce gluten content of wheat-based food products (Thompson, 2003). Furthermore, limited studies have reported nutritionally important starch fractions in food preparations, particularly in traditional food products; hence, modifying food formulations by substituting the basic ingredients with oats is a viable approach to modifying starch digestibility. In view of this, the present study investigated the dietary acceptability of oats incorporated jereesh and studied the effect of oats incorporation on different starch fractions of nutritional significance using an in vitro multi-enzyme assay.

\section{Materials and methods}

\subsection{Materials}

All food ingredients were purchased from Nesto hypermarket, Ar Rass, Saudi Arabia. Amyloglucosidase (Sigma-Aldrich, St. Louis, USA), pancreatin (Sigma-Aldrich, St. Louis, USA), invertase (Sigma-Aldrich, St. Louis, USA), glucose oxidase-peroxidase reagent kit (Randox Laboratories, Crumlin, UK) were used. All of the other chemicals were of the highest analytical purity.

\subsection{Preparation of jereesh}

Crushed wheat $(250 \mathrm{~g})$ soaked in water for 4 hours was used as main ingredient. First seasoning was prepared for which oil $(30 \mathrm{~mL})$ was heated in a pan placed on low heat $\left(<150^{\circ} \mathrm{C}\right)$. To this chopped onion $(40 \mathrm{~g})$, finely chopped garlic $(5 \mathrm{~g})$, were added and sauteed till onions turned golden brown. Then tomato paste $(30 \mathrm{~mL})$, grated carrot $(30 \mathrm{~g})$, dried lemon powder $(2.5 \mathrm{~g})$, black pepper $(5 \mathrm{~g})$ and salt $(5$ g) were added and sauteed for 2-3 min following with addition of water $(250 \mathrm{~mL})$ and soaked crushed wheat. Mixed well and allowed to cook 10 min on medium heat $\left(160-190{ }^{\circ} \mathrm{C}\right)$ till wheat became soft. To prepare oats incorporated jereesh (OJ), crushed wheat was replaced with whole oats at 10, 20 and $30 \%$ levels based on preliminary studies incorporating oats in different traditional Indian food preparations of high glycemic index (Giri et al., 2017).

\subsection{Sensory analysis}

Quantitative descriptive analysis was used to assess the sensory acceptability of oats incorporated jereesh on a scale of $0-10 \mathrm{~cm}$. The one end of the scale marked as low represented the recognition threshold, while the other end of the scale marked as high indicated the saturation threshold (Stone et al., 2008). The developed score card listed appearance, color, taste, after taste, mouthfeel and overall quality as attributes of the product. Sensory analysis was carried out by 25 graduate student panelists having completed food analysis course and familiar with the product attributes and sensory evaluation techniques. Sensory analysis was carried out in airconditioned sensory booths $\left(18 \pm 2{ }^{\circ} \mathrm{C}\right)$ lit with white fluorescent light and maintained at RH $60 \pm 5 \%$ using ultrasonic humidifier (Buerer LB88, Ulm, Germany).

The food samples were coded with three-digit random codes to avoid bias and served hot $\left(50-60^{\circ} \mathrm{C}\right)$ in small cups with disposable spoons. Along with the samples, packaged drinking water was given as palate cleanser. Panelists were asked to draw a vertical line on the scale and write the code number to indicate the perceived strength of each attribute specified on the score card. The scores for each attribute for each sample were tabulated, reflecting the panelists' decision. Finally, a mean value was calculated for each sample attribute, reflecting the panel's opinion on the perceived sensory quality and dietary acceptability. A visual representation of sensory quality in the form of sensory profile was plotted using radar plot in OriginPro 2018 software (OriginLab Corporation, MA, USA).

\subsection{Measurement starch fractions of nutritional significance and starch digestibility index}

Figure 1 depicts a summary of the methodological approach employed. Total starch (TS) and nutritionally important starch fractions namely, rapidly digestible starch (RDS), resistant starch (RS) and rapidly available glucose (RAG) were measured by the method of Englyst et al. (1992). Incubation with pancreatin, invertase and amyloglucosidase at $37{ }^{\circ} \mathrm{C}$ in $50 \mathrm{~mL}$ polypropylene capped tubes positioned in a shaking water bath was used to determine different starch fractions in freshly prepared food samples. Glass beads and guar gum were added to the tubes to simulate intestinal digestive conditions by disrupting food particles and increasing viscosity of incubation mixture, respectively. Glucose content of the reaction mixture was measured at $20 \mathrm{~min}\left(\mathrm{G}_{20}\right)$ to reflect rapidly available glucose RAG and at $120 \mathrm{~min}\left(\mathrm{G}_{120}\right)$ to measure slowly digestible starch (SDS). The tubes were then placed in boiling water to gelatinize starch and treated with $7 \mathrm{M} \mathrm{KOH}$ at $0{ }^{\circ} \mathrm{C}$ and the glucose content was measured to estimate total glucose (TG). After 120 minutes of incubation, resistant starch was characterized as the starch that remained unhydrolyzed. The amount of free glucose (FG) in the sample was measured by using acetate buffer and putting the tubes in a boiling water bath for $30 \mathrm{~min}$. Simultaneous experiments with a standard dextrose were performed in a similar manner. To account for the glucose present in enzyme solutions, a blank tube containing buffer, glass beads, and guar gum was used. The glucose levels in all of the samples were measured using a glucose oxidase - peroxidase diagnostic package.

From the values of $\mathrm{G}_{20}, \mathrm{G}_{120}, \mathrm{FG}$, and $\mathrm{TG}$, the following values for TS, RDS, SDS, and RS were determined.

Total Starc $\mathrm{s}(\mathrm{TS})=(\mathrm{TG}-\mathrm{FG}) \times 0.9$

Rapidly Digestible Starch $(\mathrm{RDS})=\left(\mathrm{G}_{20}-\mathrm{FG}\right) \times 0.9$

Slowly Digestible Starch $(\mathrm{SDS})=\left(\mathrm{G}_{120}-\mathrm{G}_{20}\right) \times 0.9$

Resistant Starch $(\mathrm{RS})=\mathrm{TS}-(\mathrm{RDS}-\mathrm{SDS}) \times 0.9$ or $\left(\mathrm{TG}-\mathrm{G}_{120}\right) \times 0.9$

Starch digestibility index and rapidly available glucose were 


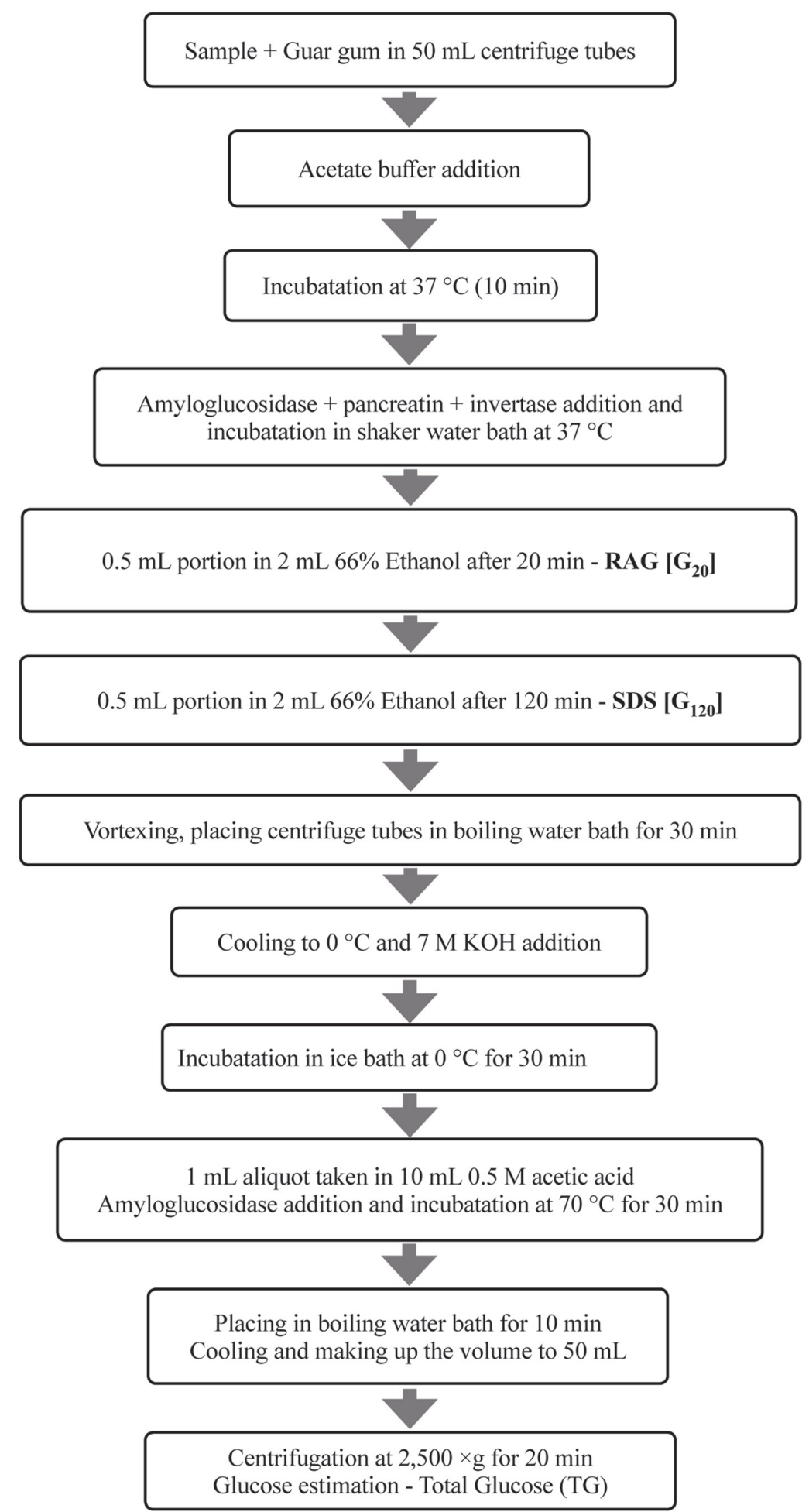

Figure 1. Schematic summary of the analytical strategy for measurement of different starch fractions.

calculated using the following formulas.

Starch Digestibility Index $(\mathrm{SDI})=\frac{\mathrm{RDS}}{\mathrm{TS}} \times 100$

Rapidly available glucose $(\mathrm{RAG})=\mathrm{FG}+\mathrm{G}_{20}$

\subsection{Statistical analysis}

The results for different starch fractions (RDS, SDS, RS), TS, SDI, and RAG are expressed as the mean \pm SD of triplicate measure- 


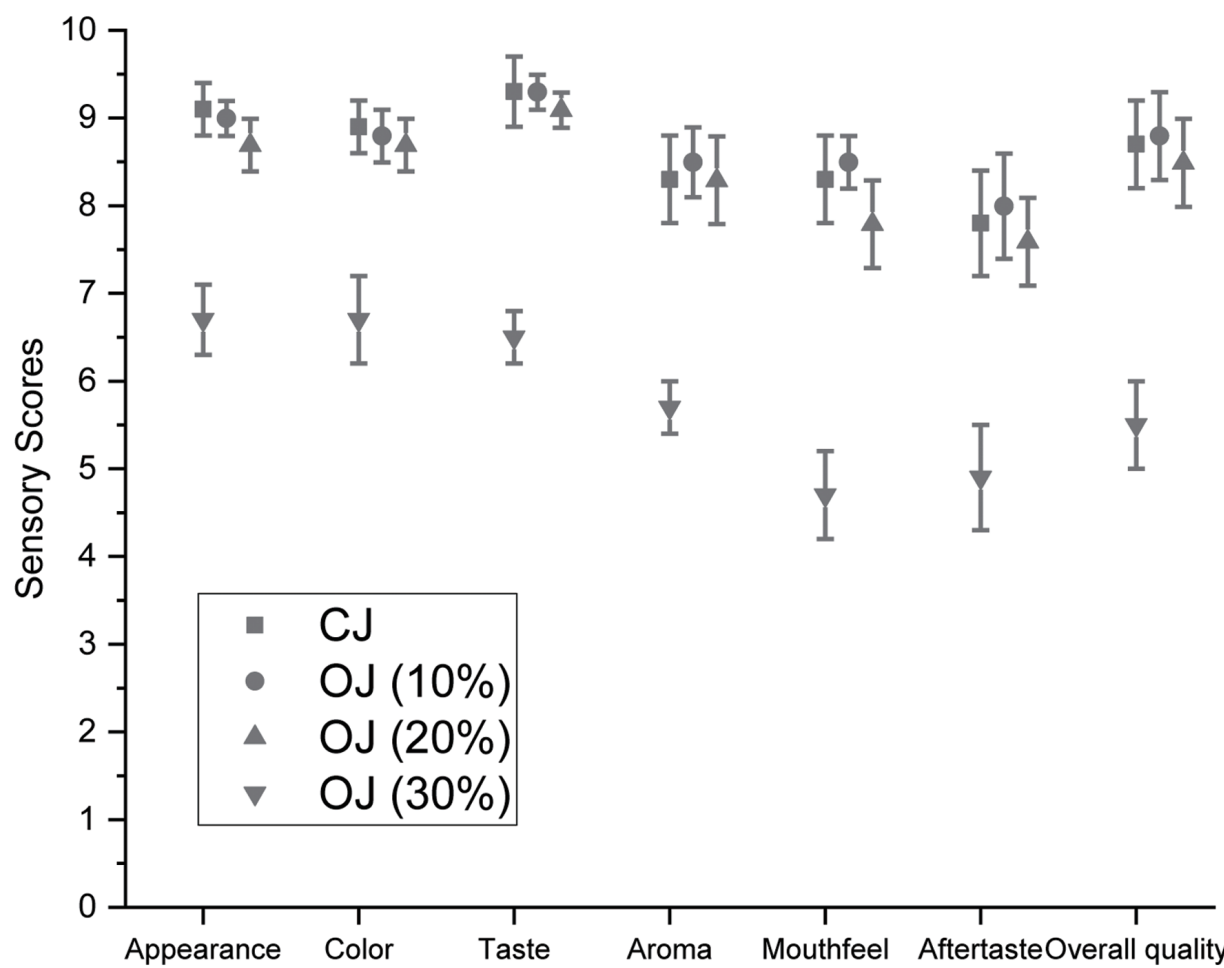

Figure 2. Sensory scores of control jereesh and oats incorporated jereesh. CJ: control jereesh, OJ (10\%): $10 \%$ oats incorporated jereesh, OJ (20\%): $20 \%$ oats incorporated jereesh, OJ (30\%): $30 \%$ oats incorporated jereesh.

ments. Using SPSS 20.0 program, data was analyzed variance. ANOVA test was used and significant differences between groups were determined using Tukey's post-hoc test at $95 \%$ confidence level. The graphs were plotted using OriginPro 2018 software (OriginLab Corporation, MA, USA).

\section{Results and discussion}

\subsection{Sensory analysis}

The study evaluated the dietary acceptability of oats incorporated jereesh - An Arabian traditional food and studied its starch digestibility characteristics using in vitro hydrolysis. In order to select the best proportion of oats to wheat replacement, a pilot study using three levels of oats replacement (10,20 and 30\%) was conducted. The sensory scores obtained are presented in Figure 2. It was found that, incorporating oats at 10 and $20 \%$ did not affect any of the sensory attributes (color, appearance, taste, aroma, mouthfeel, after taste and overall quality) significantly $(\mathrm{p}<0.05)$ compared to control product, while the product with $30 \%$ oats incorporation was not acceptable in terms of any of the sensory attributes as indicated by significantly lower sensory scores. Therefore, $20 \%$ oats incorporated jereesh was selected to study redistribution of starch fractions further. It is noteworthy that, though the product developed with $20 \%$ oats replacement was slightly viscous (sticky) due to the presence of high $\beta$-glucan in oats (Havrlentova and Kraic, 2006), its dietary acceptability was unaffected as reflected by the sensory scores (Figure 3). These findings encourage utilization of oats in traditional foods because of their high acceptability among general population and high sensory acceptability scores of oats incorpo- rated jereesh which were comparable to that of control jereesh.

\subsection{Nutritionally important starch fractions and starch digest- ibility index}

Effect of incorporation of oats on total starch (TS), rapidly digestible starch (RDS), slowly digestible starch (SDS) and resistant starch $(\mathrm{RS})$ is summarized in Table 1. A significant $(\mathrm{p}<0.05)$ reduction in total starch (TS), rapidly digestible starch (RDS) as well as resistant starch (RS) was observed in oats incorporated jereesh (OJ) compared to control jereesh (CJ). TS, RDS and RS were reduced by an extent of $3.07,0.62$ and $3.18(\mathrm{~g} / 100 \mathrm{~g}$ on as eaten basis), respectively in OJ compared to CJ. On the other hand, a significant $(\mathrm{p}<0.05)$ increase in slowly digestible starch (SDS) was seen in OJ compared CJ to an extent of $0.97 \%$. Similar trend was seen with respect to rapidly available glucose (RAG), wherein RAG was decreased from $4.84 \%$ in CJ to $3.64 \%$ in oats incorporated jereesh.

Though, there was a significant $(\mathrm{p}<0.05)$ reduction in total starch content and rapidly available glucose (RAG) in oats incorporated jereesh compared to control jereesh, no significant $(\mathrm{p}<0.05)$ differences were observed between the starch digestibility index (SDI) of CJ and OJ (Table 1).

The methods used in this study allow for precise measurement of individual starch nutritional fractions based on their responsiveness to enzymatic digestion, which is affected by the food matrix. Also, since it is important that the in vitro technique used to mimic and predict in vivo starch digestion as closely as practicable, foods were examined as they are eaten in general (Ahmed and Urooj, 2008). The study evaluated the impact of incorporating oats as an excellent source of soluble dietary fiber, on total starch, different 


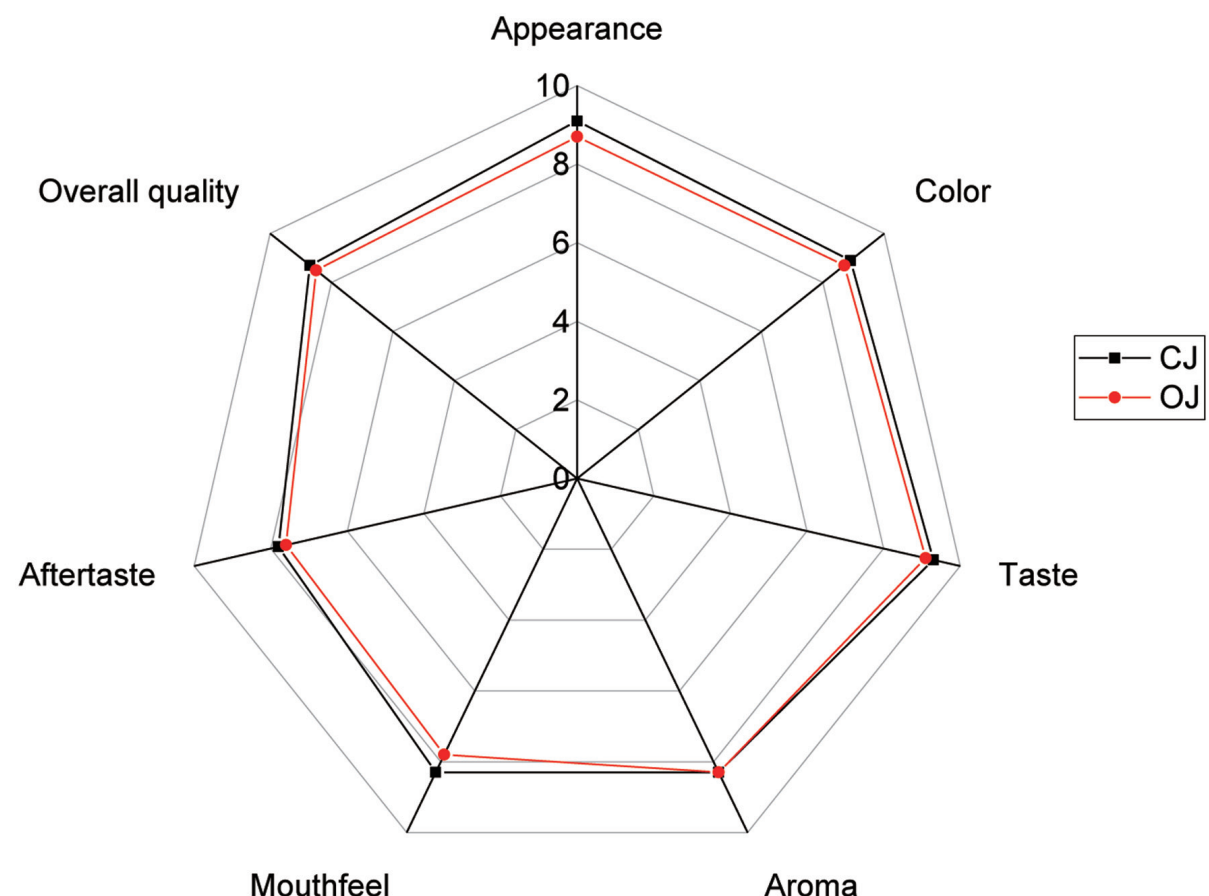

Figure 3. Sensory profile of control jereesh and oats incorporated jereesh. CJ: control jereesh, OJ: oats incorporated jereesh.

starch nutritional fractions and its digestibility in a wheat-based breakfast cereal - jereesh. Despite the fact that the developed product was viscous, its acceptability was high, and the observed rise in product's viscosity can be attributed to higher $\beta$-glucan content of oats (Havrlentova and Kraic, 2006).

As expected, incorporation oats in jereesh significantly decreased total starch content, rapidly digestible starch, resistant starch and rapidly available glucose but starch digestibility index remained unchanged. The decrease in total starch content is proportionate to starch content of the ingredients used which can be attributable to the replacement of wheat starch with $\beta$-glucan present in oats. Similar findings have been reported earlier by Brennan et al. (2008) wherein, incorporation of dietary fiber source in extruded breakfast cereal products reduced led to a significant reduction in total starch content. This observation is also in good agreement with an earlier study wherein, incorporation of oats led

Table 1. Nutritionally important starch fractions in control and oats incorporated jereesh (Mean \pm SD g/100g)

\begin{tabular}{lll}
\hline Component* & CJ & OJ \\
\hline Dry matter & 28 & 28 \\
TS & $16.64^{\mathrm{b}} \pm 0.57$ & $13.57^{\mathrm{a}} \pm 0.24$ \\
RDS & $3.38^{\mathrm{a}} \pm 0.21$ & $2.52^{\mathrm{b}} \pm 0.23$ \\
SDS & $0.46^{\mathrm{a}} \pm 0.09$ & $1.43^{\mathrm{b}} \pm 0.29$ \\
RS & $12.80^{\mathrm{b}} \pm 0.40$ & $9.62^{\mathrm{a}} \pm 0.33$ \\
RAG & $4.84^{\mathrm{b}} \pm 0.17$ & $3.64^{\mathrm{a}} \pm 0.28$ \\
SDI & $20.30^{\mathrm{a}} \pm 0.95$ & $18.54^{\mathrm{a}} \pm 1.39$
\end{tabular}

*Mean values carrying different superscript letters $a$ and $b$ in rows differ significantly ( $p \leq 0.05$ ). CJ, control jereesh; OJ, oats incorporated jereesh; TS, total starch; RDS, rapidly digestible starch; SDS, slowly digestible starch; RS, resistant starch; RAG, rapidly available glucose; SDI, starch digestibility index. to a significant reduction in the total starch content of some traditional Indian foods - idli, dosa, upma, and chapatti (Giri et al., 2017).

The involvement of several factors in food preparations, the most important of which are degree of gelatinization, cooking procedures, food source, and macronutrient composition, regulates the in vitro rate of starch digestion (Urooj and Puttraj, 1999). RAG is the sum of RDS and free sugars that are present in the food and considered useful in predicting the glycemic responses in vivo (Englyst et al., 1992; Englyst and Hudson, 1996). Therefore, a starch digestibility index of $18.54 \%$ observed in OJ aided with low RDS and RAG could be beneficial in maintaining glucose homeostasis amongst obese and glucose intolerant population.

When foods are exposed to conditions that decrease accessibility of starch molecules to enzymatic activity either by creating a physical barrier across the enzyme or by inhibiting the enzyme, the rate of starch digestibility decreases (Tester et al., 2004). It is noteworthy that incorporation oat flours in different foods products have been reported to inhibit starch digestibility, resulting in lower GI in foods (Kim and White, 2012). The results obtained in our study further substantiate the earlier findings and support the use of oats as functional ingredients in the development of novel foods products with reduced starch digestibility which is expected in various medical conditions such as diabetes, obesity, polycystic ovarian syndrome and metabolic syndrome. The findings indicate that, in food formulations, soluble fiber can influence the rate of starch digestibility to varying extent depending on several other factors such as food matrix, viscosity afforded by the soluble fibers and the amount of water used (Jenkins, 2007; Remya et al., 2017).

\section{Conclusion}

This study demonstrated the dietary acceptability of oats incorpo- 
rated jereesh; a traditional Arabic food. The observed decrease in RAG and RDS with associated increase in SDS levels might lower glycemic index of the developed product that could be beneficial for populations with impaired glucose tolerance, obesity and diabetes. The findings of this study may help in the development of tailored functional foods with altered starch digestibility characteristics. However, further research is warranted to study the effect of oats incorporation in traditional food products on glycemic index in real life situations.

\section{Acknowledgments}

None to declare.

\section{Conflict of interest}

The author declares no conflict of interest.

\section{References}

Ahmed, F., and Urooj, A. (2008). In vitro starch digestibility and nutritionally important starch fractions in processed roots and tubers. Starch/ Stärke 60: 493-499.

Al-Faris, N.A. (2017). Nutritional Evaluation of Selected Traditional Foods Commonly Consumed in Saudi Arabia. J. Food Nutr. Res. 5: 168-175.

Al-Mssallem, M.Q. (2014). The Association between the glycaemic index of some traditional saudi foods and the prevalence of diabetes in Saudi Arabia: A review article. J. Diabetes Metab. 5: 452.

Brennan, M.A., Monro, J.A., and Brennan, C.S. (2008). Effect of inclusion of soluble and insoluble fibres into extruded breakfast cereal products made with reverse screw configuration. Int. J. Food Sci. Technol. 43 2278-2288.

Butt, M.S., Tahir-Nadeem, M., Khan, M.K.I., Shabir, R. and Butt, M.S. (2008). Oat: unique among the cereals. Eur J Nutr. 47: 68-79.

Englyst, H.N., and Hudson, G.J. (1996). The classification and measurement of dietary carbohydrates. Food Chem. 57: 15-21.

Englyst, H.N., Kingman, S.M., and Cummings, J.H. (1992). Classification and measurement of nutritionally important starch fractions. Eur. J.
Clin. Nutr. 46: S33-S50.

Giri, S., Banerji, A., Lele, S.S., and Ananthanarayan, L. (2017). Starch digestibility and glycaemic index of selected Indian traditional foods: Effects of added ingredients. Int. J. Food Prop. 20: S290-S305.

Harasym, J., and Olędzki, R. (2018). The mutual correlation of glucose, starch, and beta-glucan release during microwave heating and antioxidant activity of oat water extracts. Food Biopro. Technol. 11: 874-884.

Havrlentova, M., and Kraic, J. (2006). Content of $\beta$-D-glucan in cereal grains. J. Food Nutr. Res. 45: 97-103.

Jenkins, D.J.A. (2007). The Glycemic Index: Looking Back 25 Years. Cereal Foods World 52: 50-53.

Kim, H.J., and White, P.J. (2012). In vitro digestion rate and estimated glycemic index of oat flours from typical and high $\beta$-glucan oat lines. J. Agric. Food Chem. 60: 5237-5242.

Nasib, O.M. (2003). Effect of chemical component and methods of preparation of some traditional Saudi Arabian foods on glycemic index. Thesis. King Saud University, College of Food \& Agricultural Sciences, Saudi Arabia.

Peterson, D.M. (2004). Oat- multifunctional grain. In: 7th international oat conference, Helsinki, Finland. Agri. Food Res. Rep. 51: 21-26.

Remya, R., Jyothi, A.N., and Sreekumar, J. (2017). Comparative study of RS4 type resistant starches derived from cassava and potato starches via octenyl succinylation. Starch-Stärke. 69: 1600264.

Samarakoon, E.R.J. (2020). Impact of physical modifications on starch nutritional fractions: rapidly digestible starch, slowly digestible starch and resistant starch. J. Food Bioact. 12: 106-121.

Samarakoon, E.R.J., Waduge, R., Liu, Q., Shahidi, F., and Banoub, J.H. (2020). Impact of annealing on the hierarchical structure and physicochemical properties of waxy starches of different botanical origins. Food Chem. 303: 125344

Stone, H., Sidel, J., Oliver, S., Woolsey, A., and Singleton, R.C. (2008). Sensory evaluation by quantitative descriptive analysis. Descriptive sensory analysis in practice. Food and Nutrition Press, Inc.

Tester, R.F., Karkalas, J., and Qi, X. (2004). Starch structure and digestibility Enzyme-Substrate relationship. World Poultry Sci. J. 60: 186-195.

Thompson, M.S. (2003). Oats and the gluten-free diet. J. Am. Diet. Assoc. 103: $376-379$.

Urooj, A., and Puttraj, S. (1999). Digestibility index and factors affecting rate of starch digestion in vitro in conventional food preparation. Nahrung 43: 42-47.

Wang, L.Z., and White, P.J. (1994). Structure and physicochemical properties of starches from oats with different lipid contents. Cereal Chem. 71: $443-450$ 